\title{
Search strategies for vector-like quark partners at LHC run-II
}

\author{
Thomas Flacke ${ }^{1,2, \star}$ \\ ${ }^{1}$ Center for Theoretical Physics of the Universe, Institute for Basic Science (IBS), Daejeon 34051, Korea \\ ${ }^{2}$ Department of Physics, Korea University, Seoul 136-713, Korea
}

\begin{abstract}
We present results on several new search channels and strategies for heavy vector-like quark partners or vector resonances at the LHC run-II. Run-II has sensitivity to single- and pair-produced quark partners with masses beyond $1 \mathrm{TeV}$, and for even higher masses for vector resonances. Decays of such heavy particles yield highly boosted tops, Higgses, and weak gauge bosons. At high boost, the Standard Model background of hadronic final states can be substantially suppressed when applying jet-substructure techniques. We present several case studies where the identification of hadronically decaying tops, Higgses, and/or electroweak gauge bosons allow to make new search channels competitive at run-II.
\end{abstract}

\section{Motivation and Overview}

Naturalness has for a long time been a guiding principle of theoretical particle physics. While the discovery of the last missing Standard Model SM particle - the Higgs - marks a great success of particle physics, it is difficult to understand why the Higgs mass is so much smaller than the Planck mass. Composite Higgs models provide a possible solution to this hierarchy problem [1, 2]. The main idea behind composite Higgs models is to realize the Higgs multilplet as a light bound state of a theory which becomes strongly coupled below a scale of a few TeV. The large hierarchy between the $\mathrm{TeV}$ scale and the Planck scale is realized through dimensional transmutation. If the Higgs multiplet is realized as a pseudo-Nambu-Goldstone boson (pNGB) multiplet of a global symmetry of the underlying model, a small hierarchy between the Higgs mass $m_{h}$, the electroweak scale $v$ and the decay constant $f$ of the pNGB can be realized at the price of a mild fine-tuning. From a low-energy perspective, the most minimal implementation of the composite Higgs paradigm is realized in models with $S O(5) \rightarrow S O(4)$ breaking [3]. Within such effective field theory descriptions, indirect bounds like deviations of Higgs couplings from the Standard Model (SM) prediction and electroweak precision tests yield constraints of $f \gtrsim 500-1000 \mathrm{GeV}$, depending on the precise model, the top-partner masses, and the underlying UV dynamics. Flavour physics also yields important constraints, as obtaining the observed top mass requires an extended (top-)quark sector with additional sources for flavor changing neutral currents. Partial compositeness of the top can successfully address these flavor problems as recently discussed in [4].

On the other hand, composite Higgs models can be tested directly by searching for composite resonances beyond the Higgs. Composite top-partners, scalar- or vector resonances are expected to

\footnotetext{
^e-mail: flacke@ibs.re.kr
} 
have masses at a scale $\sim g_{\text {strong }} f \lesssim 4 \pi f$ i.e. in the multi-TeV range. Stabilizing the Higgs mass and electroweak vacuum at the SM values typically requires top-partners to be even lighter, i.e. in the TeV range. It should be noted that generically, UV embeddings of composite Higgs models require an Goldstone-boson sector with additional electroweak and colored pNGBs, as underlying models with only elementary fermions and hyper-color gauge bosons cannot realize the minimal global symmetry breaking pattern $S O(5) \rightarrow S O(4)$ [5]. The additional pNGBs are likely to be the lightest BSM states in composite Higgs model UV embeddings, and they have implications for di-boson and di-top searches (c.f. [6-9]), but in this talk we focus mainly on searches for top- and other quark-partners.

\section{A sample model: the minimal composite Higgs model}

In composite Higgs models, the Higgs multiplet is realized as a pNGB multiplet which is thought of as a bound state of the underlying strongly coupled theory. The vector-like quark partners are bound states of the strongly coupled theory, which are assumed to couple linearly to elementary quarks. These couplings (as well as weakly gauging the $S U(2)_{L} \times U(1)$ in the surviving global subgroup) explicitly break the global symmetry and induce a Higgs potential, making the Higgs a pNGB. The linear couplings between the elementary quarks and their partners (which come in multiplets of the global symmetry) induce mass-mixing between each other such that the lightest mass eigenstates (which are to be identified with the SM quarks) are a linear combination of the elementary quarks and strongly coupled states and are thus termed "partially composite". The mass-mixing also induces couplings between SM quarks, their heavy quark partners, and the electroweak bosons $W, Z, h$, which are relevant for the production of heavy quark partners at the LHC and the partners' decays and branching ratios. The typical strength of these couplings is the corresponding EW (or Yukawa) coupling times a mixing angle. In these proceedings we refrain from giving the full Lagrangian and interactions used for our simulations and model implementations (they can be found in Refs.[10, 11]) and instead summarize the qualitative features relevant for LHC searches:

- Quark partners come in multiplets of the global symmetry group in which the the EW group is embedded. For example a top-partner multiplet embedded into the 5 of $S O(5)$ contains one charge $5 / 3$ partner, three charge $2 / 3$ partners and one charge $-1 / 3$ partner.

- Quark partners are charged under $S U(3)$ and can be QCD-pair produced. This production mechanism is rather model-independent as the coupling strength is fixed, and the production cross section only depends on the mass of the quark partner.

- Quark partners can be single-produced from SM quarks and EW gauge bosons. The couplings relevant for this production mechanism are generically of electroweak strength or below, but modeldependent.

- The branching ratios of quark partners are generically model-dependent. Again taking quark partners in the 5 of $S O(5)$ as an example: the charge $5 / 3$ state $X_{5 / 3}$ can only decay into $W^{+} t$ due to charge conservation while the three charge $2 / 3$ states (which we collectively denote by $T$ ) can decay into $W^{-} b, Z t$ or $h t$, and the respective branching ratios are model- and parameter dependent.

\section{Heavy quark searches at the LHC}

\subsection{A brief summary of existing LHC top partner searches}

Both ATLAS and CMS performed numerous dedicated searches for 3rd family quark partners at the LHC run-I. The searches mostly focus on QCD pair production of charge $5 / 3,2 / 3$, and $-1 / 3$ partners 
and their decay into 3rd family quarks and $W / Z / h$. Searches are performed in various final states, including all-hadronic searches (using jet-substructure techniques), single- or multi-lepton final state searches, and - for charge 5/3 partner searches - also same-sign dilepton final states. The mass bounds on 3rd family partners are $m_{X 53}>940 \mathrm{GeV}$ [12] (run-II bound), $m_{T}>700-950 \mathrm{GeV}$ [13-16] (still run-I bounds), and similar bounds for $m_{B}$ where the $m_{T}$ and $m_{B}$ bounds have a range because they depend on the $T$ and $B$ branching fractions into different final states.

Most ATLAS and CMS searches so far focussed on QCD pair produced top partners while for singly produced top partners bounds were obtained in a recast of other experimental searches in Ref. [17], which show that for typical couplings (in composite Higgs models), single-production channels yield at most comparable and mostly weaker bounds on the quark partner masses than the pair-production channels for LHC run-I. First ATLAS and CMS bounds for single-production (from run-II) are available only since very recently [18-22]. They constrain the single-production cross section times branching ratio for the final states $t h, t Z$, and $b W$ to $\sim 400 \mathrm{fb}, \sim 200 \mathrm{fb}$, and $\sim 200 \mathrm{fb}$, respectively. ${ }^{1}$

Bounds for vector-like quark partners of light quarks have been obtained in a recast from several experimental searches in Ref. [10]. The bounds from QCD pair production are generically weaker than for 3rd family quark partners. In particular a bound on the charge $5 / 3$ partner is obtained at $m_{X 53}>530 \mathrm{GeV}$. Notably, the bound on the charge $2 / 3$ partner can be even weaker $\left(M_{Q}>410 \mathrm{GeV}\right)$ if it dominantly decays into $h j$ [23]. ${ }^{2}$ Bounds on singly-produced 1st or 2nd family quark partners can be stronger, but are model-dependent.

\section{Improved search strategies for quark partners}

In this section, we discuss various improved search strategies for top- and quark partners, designed for LHC run-II.

\subsection{Search options for charge 2/3 top-partners}

In Ref. [25], we performed a comprehensive overview on search options for singly produced charge $2 / 3$ top partners which decay into $t h, t Z$, or $W b$. The pair-production and single-production cross section at $\sqrt{s}=14 \mathrm{TeV}$ and for typical values of the couplings are shown in Fig. 1, left. The branching ratios into these three final states are model-dependent, such that we analyze each channel separately. For all decay channels, hadronic decays of the $t, h, W, Z$ have larger branching ratios, but suffer from larger SM backgrounds, such that a careful analysis was necessary in order to identify the most promising set of searches. For the $T^{\prime} \rightarrow t Z$ channel, the cleanest channel would be the leptonic decay of both the top and the $Z$, but it suffers from a low branching ratio. The most promising channels are the $t_{\text {had }} Z_{l l}$ and $t_{\text {had }} Z_{\text {inv }}$ channel, where the first one is more standard. The $Z_{\text {inv }}$ channel benefits from a lager branching ratio but has more SM background. We use jet-substructure techniques (in particular the template overlap method) for top-tagging, and a forward jet tag for both channels, a standard $Z_{l l}$ reconstruction for the leptonic decay and a hard MET cut for the invisible decay. In our analysis we show that both channels have comparable prospects for discovery if $M_{T^{\prime}} \lesssim 1 \mathrm{TeV}$, but for larger $M_{T^{\prime}}$, the prospects for the $Z_{\text {inv }}$ become more favorable, as a hard MET cut reduces the SM background very effectively. For details, c.f. [25, 26].

We also performed analyses for various $t h$ and $W b$ decay channels and find the best performance for the $t_{\text {had }} h_{b b}$ and $W_{\text {lep }} b$ channels. Fig. 2 shows a projection of the production cross sections of $T^{\prime}$

\footnotetext{
${ }^{1}$ The bound on the cross section depends on the mass of the resonance, and the values are only given for orientation. For more precise information see Refs. [18-22].

${ }^{2}$ The sensitivity on this di-Higgs, di-jet channel can be substantially improved at LHC run-II [24].
} 

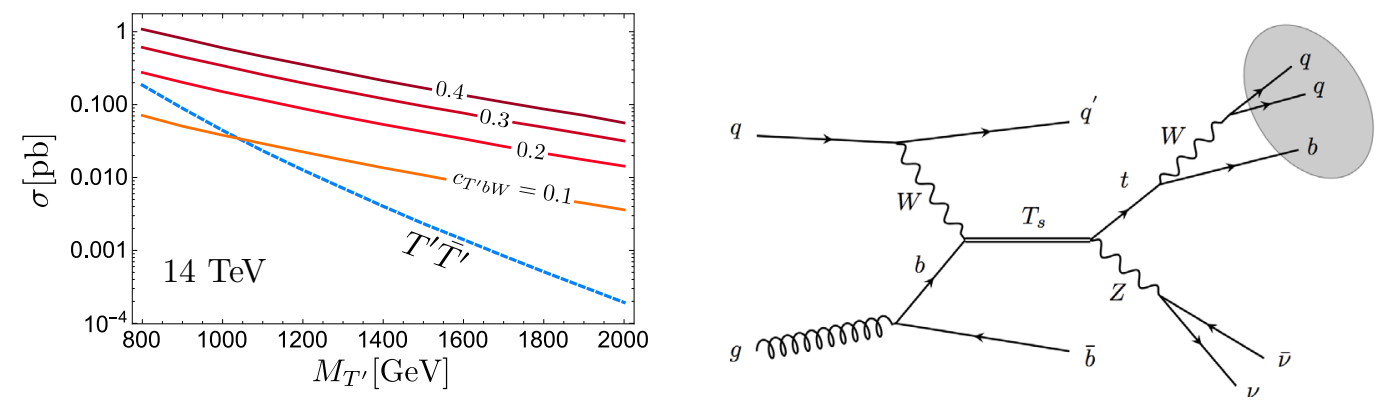

Figure 1. Left: Single- and pair-production cross section of top partners at $\sqrt{s}=14 \mathrm{TeV}$. Right: Singleproduction of a top partner with subsequent decay to $t_{\text {had }} Z_{\text {inv }}$.
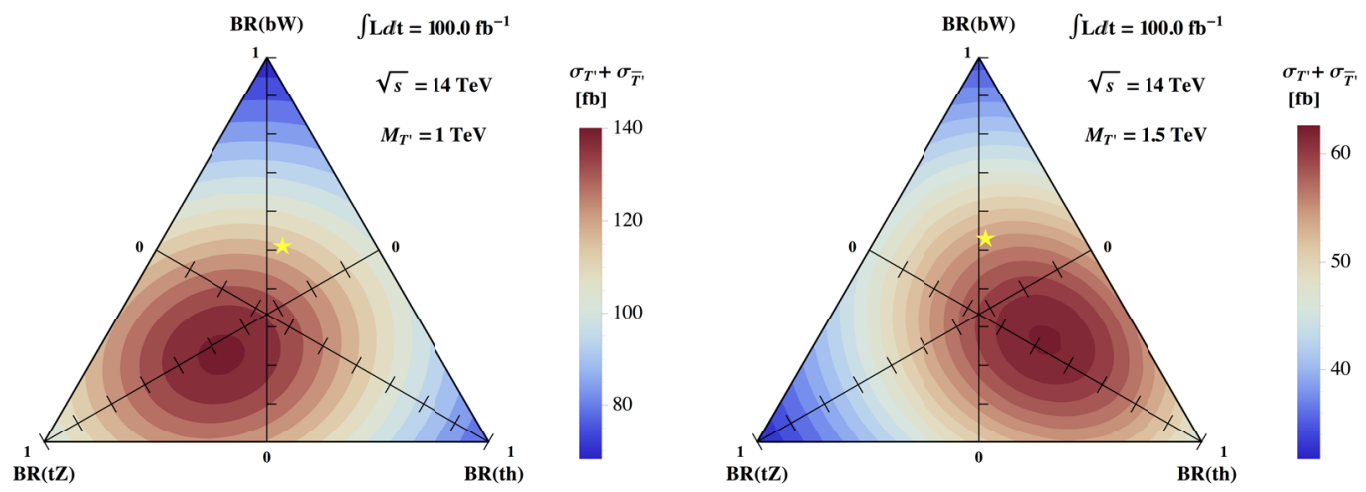

Figure 2. Expected discovery reach for a $T^{\prime}$ with mass of $1 \mathrm{TeV}$ (left) and $1.5 \mathrm{TeV}$ (right) in terms of $T^{\prime}$ production cross section for the $\mathrm{LHC}$ at $14 \mathrm{TeV}$ with $100 \mathrm{fb}^{-1}$ of data.

at which a $T^{\prime}$ with a mass of $1 \mathrm{TeV}$ (left) or $1.5 \mathrm{TeV}$ (right) can be discovered with an integrated luminosity of $100 \mathrm{fb}^{-1}$. The analogous figures for exclusion reach can be found in Ref.[25]. For an analysis of the $X_{5 / 3}$ single-production channel with semi-leptonic decay of $t W$ c.f. Ref. [11].

\subsection{Di-Higgs signatures from composite quark partners}

As another example, we studied pair production of a quark partner $U_{h}$ which decays into $u h .^{3}$ For definiteness we chose the vector-like quark to be the partner of the up-quark. Fig. 3 (left) shows the QCD pair production cross section of the up-partner, while the topology of the event is displayed in Fig. 3 (left). As can be seen, this decay can lead to a substantial cross section for a di-higgs final state

\footnotetext{
${ }^{3}$ For the purpose of this study, we assume a $u h$ branching ratio of $100 \%$. C.f. Ref.[24] for a model which yields such a dominant $q$ h decay.
} 

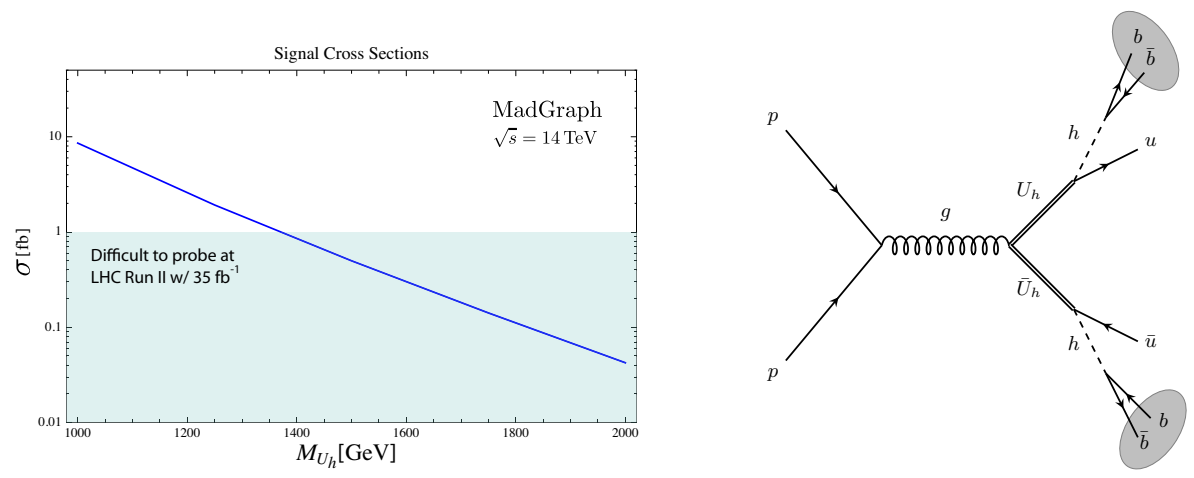

Figure 3. Left: QCD pair-production of quark partners at $\sqrt{s}=14 \mathrm{TeV}$. Right: parameter-independent topology of di-Higgs - di-quark production in models with composite light quark partners.

with two additional quarks. Nevertheless, as has been shown in Ref. [23], this di-Higgs final state is not well covered by early LHC searches, partially because the Higgses are strongly boosted, but early LHC searches did not provide information on the $p_{T}$ distribution of the Higgses in di-higgs searches.

We designed a search strategy in order to maximize the sensitivity to the $q q h_{b b} h_{b b}$ final state (with boosted Higgses). Again, we use jet-substructure techniques to identify the boosted Higgses. Furthermore, we use that the invariant mass of the two $q h$ pairs are identical (if paired correctly) which can be checked on an event-by-event basis, and which also yields a good mass resolution for the determination of $M_{U_{h}}$. We find that with a luminosity of $35 \mathrm{fb}^{-1}$, a light quark partner with $100 \%$ BR into $q h$ can be discovered at a mass of above $1 \mathrm{TeV}$, with an expected $S / \sqrt{B}=7.5$ at $M_{U_{h}}=1 \mathrm{TeV}$ and $S / \sqrt{B}=3.8$ at $M_{U_{h}}=1.2 \mathrm{TeV}$, implying a well increased sensitivity for this most "elusive" channel.

The above analysis has been performed at leading order, only, and due to the large number of quarks in the all-hadronic final state, an NLO analysis of the production cross section and kinematical distributions are desirable in order to obtain more reliable results. A joint group effort in this direction has been started in Les Houches 2015 [27], followed by first published results [28].

\section{Outlook on searches for vector resonances with decays to top partners}

In the last section, we discussed production and decay of quark- or top-partners in absence of other composite resonances. However, composite Higgs models are expected to also contain vector resonances. If they are present, but heavier than the top-partners, two interesting effects occur. The interaction between the vector- and top partners arises from the strong sector, and is expected to dominate over the couplings of vector resonances to the SM particles. Thus, the vector resonances dominantly decay to top and top-partner (if $m_{\rho}<2 m_{T^{\prime}}$ ) or into two top partners (if $m_{\rho}>2 m_{T^{\prime}}$ ). This on the one hand reduces the bounds on vector resonance searches in SM decay channels, and on the other hand, it provides an additional, resonant, production mode for top-partner pairs or a top-top-partner final state, which lead to 3- or 4-body final states from the vector-resonance decays. Such channels provide interesting signatures for future collider searches of vector resonance and top-partner searches. 


\section{Conclusions}

Motivated by composite Higgs models, we presented several new search strategies for vector-like toppartners and other quark-partners for LHC run II. We found that jet-substructure techniques strongly increase the sensitivity in these searches, because the discrimination power to background for the decay products from heavy vector-like quarks (in particular tops, $W, Z$ bosons and Higgses) can be substantially increased as the decay products are strongly boosted. We also pointed out that the interplay of vector resonances and vector-like quarks can alter the interpretation of existing searches and promises interesting signatures to be explored in run-II.

Note added: After this talk was given, we published a first work on identifying $Z^{\prime}$ resonances in decays to $t T^{\prime}$ in the $\bar{t} t Z$ final state, which quantifies our statements on the interplay of vector resonances and top $=$ partners [29].

\section{Acknowledgements}

I would like to thank the ISMD organizers for setting up this extraordinary conference. My work was supported by the Basic Science Research Program through the National Research Foundation of Korea (NRF) funded by the ministry of Education, Science and Technology (No. 2013R1A1A1062597), by the France-Korea Particle Physics Lab (FKPPL), and by IBS under the project code, IBS-R018-D1.

\section{References}

[1] D.B. Kaplan, H. Georgi, Phys. Lett. B136, 183 (1984)

[2] D.B. Kaplan, H. Georgi, S. Dimopoulos, Phys. Lett. B136, 187 (1984)

[3] K. Agashe, R. Contino, A. Pomarol, Nucl. Phys. B719, 165 (2005), hep-ph/0412089

[4] G. Cacciapaglia, H. Cai, T. Flacke, S.J. Lee, A. Parolini, H. Serôdio, JHEP 06, 085 (2015), 1501.03818

[5] G. Ferretti, D. Karateev, JHEP 03, 077 (2014), 1312 . 5330

[6] H. Cai, T. Flacke, M. Lespinasse (2015), 1512.04508

[7] A. Belyaev, G. Cacciapaglia, H. Cai, T. Flacke, A. Parolini, H. Serôdio, Phys. Rev. D94, 015004 (2016), 1512.07242

[8] G. Cacciapaglia, H. Cai, A. Deandrea, T. Flacke, S.J. Lee, A. Parolini, JHEP 11, 201 (2015), 1507.02283

[9] A. Belyaev, G. Cacciapaglia, H. Cai, G. Ferretti, T. Flacke, A. Parolini, H. Serôdio (2016), 1610.06591

[10] C. Delaunay, T. Flacke, J. Gonzalez-Fraile, S.J. Lee, G. Panico, G. Perez, JHEP 02, 055 (2014), 1311.2072

[11] M. Backović, T. Flacke, S.J. Lee, G. Perez, JHEP 09, 022 (2015), 1409. 0409

[12] CMS (2015), CMS-PAS-B2G-15-006

[13] G. Aad et al. (ATLAS), JHEP 10, 150 (2015), 1504.04605

[14] G. Aad et al. (ATLAS), JHEP 11, 104 (2014), 1409. 5500

[15] G. Aad et al. (ATLAS), JHEP 08, 105 (2015), 1505.04306

[16] V. Khachatryan et al. (CMS), Phys. Rev. D93, 012003 (2016), 1509.04177

[17] O. Matsedonskyi, G. Panico, A. Wulzer, JHEP 12, 097 (2014), 1409. 0100

[18] CMS (2015), CMS-PAS-B2G-15-008 
[19] CMS (2016), CMS-PAS-B2G-16-001

[20] CMS (2016), CMS-PAS-B2G-16-005

[21] CMS (2016), CMS-PAS-B2G-16-006

[22] ATLAS (2016), ATLAS-CONF-2016-072

[23] T. Flacke, J.H. Kim, S.J. Lee, S.H. Lim, JHEP 05, 123 (2014), 1312 . 5316

[24] M. Backović, T. Flacke, J.H. Kim, S.J. Lee, JHEP 04, 082 (2015), 1410. 8131

[25] M. Backović, T. Flacke, J.H. Kim, S.J. Lee, JHEP 04, 014 (2016), 1507.06568

[26] M. Backović, T. Flacke, J.H. Kim, S.J. Lee, Phys. Rev. D92, 011701 (2015), 1501.07456

[27] G. Brooijmans et al., Les Houches 2015: Physics at TeV colliders - new physics working group report, in 9th Les Houches Workshop on Physics at TeV Colliders (PhysTeV 2015) Les Houches, France, June 1-19, 2015 (2016), 1605.02684

[28] B. Fuks, H.S. Shao (2016), 1610.04622

[29] M. Backović, T. Flacke, B. Jain, S.J. Lee (2016), 1610.08810 